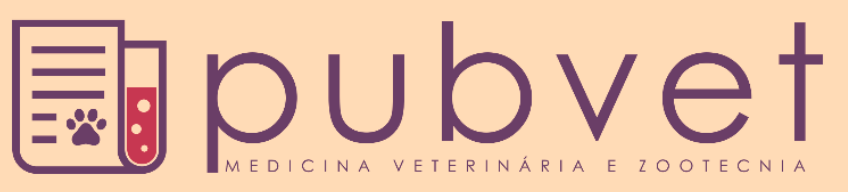

https://doi.org/10.31533/pubvet.v14n3a525.1-6

\title{
Perfil sociodemográfico da leishmaniose tegumentar americana em Almenara - Minas Gerais
}

\author{
Djully Borges da Silva Araújo ${ }^{1}$, Ednardo de Souza Nascimento ${ }^{2}$, Viviane Amaral Toledo \\ Coelho $^{3 \bullet}$, Carla Giselly de Souza ${ }^{4 *}$, Mônica Cecília Santana Pereira ${ }^{3 \bullet}$ \\ ${ }^{I}$ Bacharel em Farmácia. Alfa - Faculdade de Almenara, Almenara, Minas Gerais, Brasil. \\ ${ }^{2}$ Mestre. Alfa - Faculdade de Almenara, Almenara, Minas Gerais, Brasil. \\ ${ }^{3}$ Doutora. Alfa - Faculdade de Almenara, Almenara, Minas Gerais, Brasil. \\ ${ }^{4}$ Doutora. UFRPE - Universidade Federal Rural de Pernambuco, Recife, Pernambuco, Brasil. \\ Autora para correspondência: carlaxlsouza@yahoo.com.br
}

\begin{abstract}
Resumo. Objetivo: caracterizar o perfil sócio demográfico da leishmaniose tegumentar americana no município de Almenara - MG entre os anos de 2016-2018, considerando-se dados da notificação oficial fornecidos pelo setor de vigilância do município. Metodologia: um estudo teórico embasado em pesquisas bibliográficas referentes aos anos de 2001 á 2016, concomitantemente com um estudo prático realizado na cidade de Almenara - MG, a partir de análises de dados relativos aos anos de 2016-2018. Resultados: Ao analisarmos as ocorrências como um todo, podemos verificar que no total foram registrados 48 casos da doença. Onde, a prevalência maior de casos ocorreu na zona rural, com 77,08\% das notificações. A escolaridade chama a atenção, pois, dos 48 casos registrados, 97,92\% ou eram analfabetos, ou tinham o ensino fundamental incompleto ou ignorado. No presente trabalho $77,08 \%$ dos casos notificados foram em pacientes do sexo masculinos. $\mathrm{Na}$ literatura não há relatos que apontem a relevância desses dados. Conclusões: Sendo de difícil diagnóstico, a leishmaniose pode ser confundida com outras doenças cutâneas, retardando assim o início do tratamento, e agravando a doença. Os dados analisados são preocupantes, pois a cada ano explorado houve um crescimento considerável da doença, onde os homens foram mais acometidos do que as mulheres, a prevalência maior ocorreu na zona rural e a baixa escolaridade teve um número bastante relevante.
\end{abstract}

Palavras-chave: Transmissão, diagnóstico, epidemiologia, tratamento farmacológico

\section{Socio-demographic profile of the American cutaneous leishmaniasis in Almenara - Minas Gerais}

\begin{abstract}
Objective: To characterize the socio-demographic profile of the ATL in the municipality of Almenara - MG between 2016-2018, considering official notification data provided by the municipal surveillance sector. Methodology: a theoretical study based on bibliographic research from 2001 to 2016, concurrently with a practical study conducted in the city of Almenara - MG, from data analysis for the years 2016-2018. Results: When analyzing the occurrences as a whole, we can verify that in total 48 cases of the disease were registered. Where, the highest prevalence of cases occurred in rural areas, with $77.08 \%$ of notifications. Schooling draws attention because of the 48 cases recorded, $97.92 \%$ were either illiterate or had incomplete or ignored elementary school. In the present study, $77.08 \%$ of the reported cases were male patients. There are no reports in the literature indicating the relevance of these data. Conclusions: Being difficult to diagnose, leishmaniasis can be confused with other skin diseases, thus delaying the initiation of treatment and aggravating the disease. The data analyzed are worrying, because each year explored there was a considerable growth of the
\end{abstract}


disease, where men were more affected than women, the higher prevalence occurred in rural areas and low education had a very relevant number.

Keywords: Transmission, diagnosis, epidemiology, fharmacological treatment

\title{
Perfil sociodemográfico de la leishmaniasis cutánea americana en Almenara - Minas Gerais
}

\begin{abstract}
Resumen. Objetivo: caracterizar el perfil sociodemográfico de la leishmaniose cutanea americana en el municipio de Almenara - MG entre 2016-2018, considerando los datos de notificación oficial proporcionados por el sector de vigilancia municipal. Metodología: un estudio teórico basado en la investigación bibliográfica de 2001 a 2016, junto con un estudio práctico realizado en la ciudad de Almenara - MG, a partir del análisis de datos para los años 2016-2018. Resultados: Al analizar las ocurrencias en su conjunto, podemos verificar que en total se registraron 48 casos de la enfermedad. Donde, la mayor prevalencia de casos ocurrió en áreas rurales, con $77.08 \%$ de notificaciones. La escolarización llama la atención debido a los 48 casos registrados, el $97.92 \%$ eran analfabetos o tenían curso de primaria incompleta o ignorada. En el presente estudio, el $77.08 \%$ de los casos reportados fueron pacientes masculinos. No hay informes en la literatura que indiquen la relevancia de estos datos. Conclusiones: al ser difícil de diagnosticar, la leishmaniasis puede confundirse con otras enfermedades de la piel, lo que retrasa el inicio del tratamiento y agrava la enfermedad. Los datos analizados son preocupantes, porque a cada año explorado hubo un crecimiento considerable de la enfermedad, donde los hombres se vieron más afectados que las mujeres, la mayor prevalencia se produjo en las zonas rurales y la baja educación tuvo un número muy relevante.
\end{abstract}

Palabras clave: Transmisión, diagnóstico, epidemiología, tratamiento farmacológico

\section{Introdução}

A Leishmaniose é uma doença complexa e disseminada pelo mundo, que pode acometer regiões distintas dos tecidos parasitados e por consequência exibe diferentes formas clínicas. Pode ser considerada uma doença negligenciada, pois está associada a má nutrição, condições precárias de moradia, baixa escolarização e escassez de recursos para sobrevivência, além de demonstrar peculiaridade com áreas de desmatamento, regiões com urbanizações recentes ou com processos migratórios (Oliveira \& Fernandes, 2014). A leishmaniose apresenta-se nas formas, tegumentar (LTA) cutânea e visceral (LV), sendo na América latina conhecida como Calazar (Lucena \& Medeiros, 2018). É uma doença parasitaria grave, causado pelo agente etiológico o protozoário Leishamania, pertencentes ao complexo L. denovani e L. chagasi, que pode levar o homem a morte (Gontijo \& Melo, 2004). Esta zoonose é transmitida principalmente pelo mosquito Lutzomyia longipalpis, onde, o cão é o principal reservatório urbano do parasita, com importante papel na transmissão e epidemiologia da doença. Contudo, roedores, canídeos, marsupiais e primatas são exemplos de hospedeiros desse parasito, por conta disso, é classificada como zoonose (Lucena \& Medeiros, 2018). No Brasil, sua transmissão acomete todas as idades, principalmente crianças menores de 10 anos em áreas endêmicas, para LV e LTA (Gontijo \& Melo, 2004). A maior taxa de deteç̧ão da doença, encontra-se nas regiões CentroOeste e Nordeste (Pimenta et al., 2007). Em Minas Gerais foi reportada uma média anual de 10,5 novos casos de LV por 100.000 habitantes entre os anos 90 e 2000 (Miranda et al., 2011).

A cidade de Almenara - MG, localizada no Vale do Jequitinhonha é endêmica para LTA e nos últimos anos tem aumentado à ocorrência de casos novos LV, inclusive com óbitos. Tornando assim um problema grave de saúde pública (Miranda et al., 2011). Esta pesquisa objetivou caracterizar o perfil sócio demográfico da LTA no município de Almenara - MG, entre os anos de 2016-2018.

\section{Metodologia}

Trata-se de um trabalho descritivo e exploratório. Realizaram-se pesquisas bibliográficas (leitura e seleção de artigos) parte de um projeto de pesquisa que revela explicitamente o universo de 
contribuições científicas de autores sobre um tema específico. A pesquisa foi desenvolvida usando critérios de exclusão e aceitação de artigos publicados no período compreendido entre os anos de 2001 a 2016. Foi utilizado um total de 17 artigos e três livros com busca em artigos e publicações recentes (Scielo, Periódicos da Capes, revistas da área da saúde e Google Acadêmico), especialmente de pesquisas realizadas na região, além de análise de documentos oficiais, usando as palavras-chave: Transmissão, diagnóstico, epidemiologia, tratamento farmacológico.

Em seguida foi realizado um estudo prático, onde foram explorados e descritos dados referentes ao perfil sócio demográfico da Leishmaniose Tegumentar Americana no município de Almenara, Minas Gerais. Foram analisadas fichas de dados registrados no Sistema de Informações de Agravos de Notificação (SINAN), disponibilizados no site do DATASUS no período de 2016 a 2018 e análise documental de leis vigentes, federais, estaduais e do município de Almenara, Minas Gerais. Para a caracterização e ordenação dos dados foram utilizadas as seguintes variáveis: idade, sexo, escolaridade, raça/cor, endereço (zona rural ou urbana) e forma clínica da doença.

Por se tratar de uma análise fundamentada em banco de dados secundários e de domínio público, o estudo não foi encaminhado para apreciação de um Comitê de Ética em Pesquisa, mas ressalta-se que foram tomados os cuidados éticos que preceituam a Resolução 466/12, do Conselho Nacional de Saúde (BRASIL 2013).

\section{Resultados e discussão}

As leishmanioses são constitutivas de um importante grupo de doenças parasitárias que afligem a humanidade há vários séculos, sendo elas incluídas no leque das doenças negligenciadas. Relatos históricos indicam a sua existência desde tempos remotos na Grécia antiga (LV) bem como em países asiáticos como China, Bangladesh e outros para a LTA, sendo os processos migratórios responsáveis pela sua disseminação geográfica (BRASIL, 2004).

No Brasil, a LTA é considerada uma zoonose de difícil controle e ampla distribuição, sendo encontrada nas cinco regiões do país. Originalmente rural, a doença atualmente predomina em áreas urbanas, tendo essa modificação ocorrida principalmente devido aos processos de migração ruralurbana, mas também pelas condições sociais e econômicas precárias na população (Ursine et al., 2016).

O município de Almenara, Minas Gerais está situado na região do baixo Jequitinhonha e conta atualmente com a cobertura populacional de 96,6 \% com Estratégia de Saúde da Família (ESF). Desde o ano de 2005, a ESF assumiu os casos de leishmaniose, a partir de uma ação de descentralização. Deste modo, os bairros com cobertura, ficaram responsáveis pelo diagnóstico e tratamento dos casos, excetuando surtos reacionais de maior complexidade que são encaminhados ao serviço estadual de referência.

A LTA observa-se semelhantemente a outros estados brasileiros, a sua expansão no Estado de Minas Gerais(Almeida et al., 2010; Gontijo \& Melo, 2004; Silva \& Braga, 2010). Dados da SESMG (Secretaria de Estado de Saúde de Minas Gerais) apontam que houve a notificação 7.788 casos confirmados de LTA humana entre os anos de 2010 a 2015, com uma taxa de letalidade de 9,7\% neste período (SESMG, 2016). Destacando-se de acordo com Miranda, (2011), os municípios de Belo Horizonte, Montes Claros, Ribeirão das Neves, Janaúba, Santa Luzia e Paracatu representam cerca de 56\% das notificações. Sendo que, dentre esses, o município de Belo Horizonte é o que apresenta a maior incidência de LV humana e uma alta prevalência de LTA canina (FNS, 2010; Jesus \& Araújo, 2007; Santos et al., 2005). Além disso, outro dado relevante é a letalidade de 13,5\% observada por neste município, entre os anos de 1994 a 2011 (Ursine et al., 2016).

Os dados aqui apresentados foram coletados no SINAN, compreendendo o período de 2016-2018. Sendo assim, serão apresentados tomando como referência a região geográfica a que pertence o indivíduo, circunscrito ao município de Almenara.

A Tabela 1 apresenta o número de casos de LTA dos anos de 2016, 2017 e 2018. No ano de 2016 foram registrados 10 casos da doença, sendo uma maior incidência no sexo masculino, com $70 \%$ casos. Dentre esses números, a maioria 30\%, tinha entre 30-45 anos de idade. Com relação a escolaridade, a maior parte $40 \%$ tinham a escolaridade ignorada. 
Além disso, dos casos registrados na cidade de Almenara-Mg $40 \%$ residiam na zona urbana e $60 \%$ na zona rural. Desses, $50 \%$ foram declarados de raça branca, 20\% parda, 20\% pretas e $10 \%$ tiveram a raça ignorada. As formas clínicas encontradas foram $100 \%$ lesões, e nenhum caso de cicatriz cutânea.

Tabela 1. Casos de leishmaniose tegumentar americana, segundo as variáveis sexo, idade, escolaridade, endereço (rural/urbano), raça/cor e forma clínica. Almenara, MG, 2016, 2017 e 2018.

\begin{tabular}{|c|c|c|c|c|c|c|}
\hline \multirow{2}{*}{ Variável } & \multicolumn{2}{|c|}{2016} & \multicolumn{2}{|c|}{2017} & \multicolumn{2}{|c|}{2018} \\
\hline & $\mathrm{n}$ & $\%$ & $\mathrm{n}$ & $\%$ & $\mathrm{n}$ & $\%$ \\
\hline \multicolumn{7}{|l|}{$\overline{\text { Sexo }}$} \\
\hline Masculino & 7 & $70 \%$ & 5 & $71,42 \%$ & 25 & $80,64 \%$ \\
\hline Feminino & 3 & $30 \%$ & 2 & $28,57 \%$ & 6 & $19,35 \%$ \\
\hline \multicolumn{7}{|l|}{ Idade } \\
\hline $0-15$ & 2 & $20 \%$ & 1 & $14,28 \%$ & 8 & $25,80 \%$ \\
\hline $15-30$ & 2 & $20 \%$ & 4 & $57,14 \%$ & 2 & $6,45 \%$ \\
\hline $30-45$ & 30 & $30 \%$ & 0 & $0 \%$ & 11 & $35,48 \%$ \\
\hline $45-60$ & 10 & $10 \%$ & 1 & $14,28 \%$ & 1 & $3,22 \%$ \\
\hline $60-75$ & 20 & $20 \%$ & 1 & $14,28 \%$ & 6 & $19,35 \%$ \\
\hline \multicolumn{7}{|l|}{ Endereço (Rural/Urbano) } \\
\hline Urbano & 4 & $40 \%$ & 3 & $42,85 \%$ & 4 & $12,91 \%$ \\
\hline Rural & 6 & $60 \%$ & 4 & $57,14 \%$ & 27 & $87,09 \%$ \\
\hline \multicolumn{7}{|l|}{ Escolaridade } \\
\hline Analfabeto & 1 & $10 \%$ & 0 & $0 \%$ & 0 & $0 \%$ \\
\hline EF incompleto $\left(1^{\circ}\right.$ a $5^{\circ}$ ano $)$ & 3 & $30 \%$ & 5 & $42,85 \%$ & 27 & $87,09 \%$ \\
\hline $\mathrm{EF}$ incompleto $\left(6^{\circ}\right.$ a $9^{\circ}$ ano $)$ & 1 & $10 \%$ & 2 & $28,57 \%$ & 4 & $12,91 \%$ \\
\hline EF completo & 1 & $10 \%$ & 0 & $0 \%$ & 0 & $0 \%$ \\
\hline Ignorado & 4 & $40 \%$ & 0 & $0 \%$ & 0 & $0 \%$ \\
\hline \multicolumn{7}{|l|}{ Raça/cor } \\
\hline Branca & 5 & $50 \%$ & 2 & $28,57 \%$ & 10 & $32,25 \%$ \\
\hline Parda & 2 & $20 \%$ & 4 & $57,14 \%$ & 20 & $64,51 \%$ \\
\hline Preta & 2 & $20 \%$ & 1 & $20 \%$ & 1 & $3,22 \%$ \\
\hline Ignorado & 1 & $10 \%$ & 0 & $0 \%$ & 0 & $0 \%$ \\
\hline \multicolumn{7}{|l|}{ Forma clínica } \\
\hline Lesão & 10 & $100 \%$ & 7 & $100 \%$ & 31 & $100 \%$ \\
\hline Visceral & 0 & $0 \%$ & 0 & $0 \%$ & 0 & $0 \%$ \\
\hline
\end{tabular}

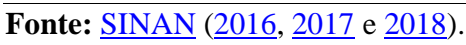

As notificações do ano de 2017, e demonstra uma queda no número de doentes de LTA em relação ao ano de 2016, registrando sete casos da doença. Mais uma vez, ocorreu uma maior incidência para o sexo masculino, contando com $71,42 \%$ ocorrências. No quesito idade, também houve queda. Para menores de 15 anos foi registrado apenas 1 caso, 14,28\%. Na faixa etária 15-30 anos ocorreu a maior parte dos casos, $57,14 \%$, nenhum caso ocorreu na faixa etária de 30-45 anos, e 14,28\% ocorreu entre as idades $45-60$ e 14,28\% entre 60-75 anos.

$\mathrm{Na}$ questão escolaridade tinham $42,85 \%$ tinham o ensino fundamental incompleto ( $1^{\circ}$ a $5^{\circ}$ ano). $\mathrm{Na}$ questão endereço residencial $57,14 \%$ eram provenientes da zona rural, estando entre esses doentes, à maioria $57,14 \%$ da raça parda. Apresentando $100 \%$ da forma clínica de lesão e nenhuma cicatriz cutânea.

Para os casos do ano de 2018, o número de acometidos voltou a crescer, registrando-se 31 casos da doença. A maior prevalência para o sexo masculino manteve-se, contando com 80,64\% das ocorrências. $\mathrm{Na}$ variável idade, a maioria dos casos 35,48\% ocorreu na idade entre 30-45 anos. No quesito escolaridade, para o ano de 2018, não foram registrados casos da doença em analfabetos. Entretanto, $87,09 \%$ dos casos se deram em pessoas com o ensino fundamental incompleto ( $1^{\circ}$ ao $5^{\circ}$ ano). Com relação à residência dos acometidos pela LTA $87,09 \%$ dos casos foram provindos da zona rural. Destes, $64,51 \%$ eram da raça parda. No que diz respeito à forma clínica, todos os casos se referiam a lesões. 
Ao analisarmos as ocorrências como um todo, podemos verificar que no total foram registrados 48 casos da doença. Onde, a prevalência maior de casos ocorreu na zona rural, com 77,08\% das notificações. Este perfil de transmissão corresponde ao que descreve o Manual de Vigilância Epidemiológica da LTA, do Ministério da Saúde, que salienta a existência de três padrões epidemiológicos evidentes no Brasil, sendo eles: silvestre, corroborando os dados encontrados, ocupacional e lazer, relacionados aos relatos de infecção por ações laborais e peri-urbano, especialmente em áreas de colonização (BRASIL, 2010). Este último, por sua vez, está diretamente associado a condições econômicas precárias, além de falta de saneamento, agravado pela migração de indivíduos da zona rural para as periferias dos centros urbanos (Basano \& Camargo, 2004).

Santos et al. (2005) salientam que aspectos relacionados às recentes alterações ambientais, podem estar associados à migração e busca de adaptabilidade do vetor. Ressalta que essas mudanças propiciam a presença do mosquito em ambientes peri-urbanos. Em contrapartida o estudo realizado por Rey et al. (2005) em crianças hospitalizadas de área endêmica, dispõe que a leishmaniose ocorre mais em áreas urbanas. O que pode ser explicado pela migração de famílias empobrecidas de camponeses, que trazem cães infectados.

A escolaridade chama a atenção, pois, dos 48 casos registrados, 97,92\% ou eram analfabetos, ou tinham o ensino fundamental incompleto ou ignorado, fato preocupante, uma vez que essa questão é importante para realizar-se a educação em saúde da população acometida pela doença, o que pode dificultar a captação das orientações sobre os cuidados necessários e tratamento. Segundo Basano \& Camargo (2004), a LTA possui aspectos geográficos, climáticos e sociais diferenciados. Almeida et al. (2010) relatam que a associação da LTA a questões socioeconômicas é uma questão que precisa ser mais bem explorada, visto que grupos populacionais de características socioeconômicas semelhantes podem possuir perfis epidemiológicos diversificados pelo fato de se localizarem em lugares diferentes e apresentarem hábitos distintos (Ursine et al., 2016). Ademais, as variáveis idade e raça/cor, os números foram bastante inconstantes. Na literatura não há relatos que apontem a relevância desses dados. Recentemente, alguns trabalhos, tem demonstrado que a infecção também está presente entre as classes com média e alta renda. Estes estudos chamam atenção para a possibilidade de uma mudança no padrão de ocorrência da doença ao revelar uma incidência significativa da LTA em áreas com boas condições socioeconômicas (Ursine et al., 2016). De acordo Guerra et al. (2003) a invasão do sexo masculino aos habitats naturais dos transmissores, gera uma maior ocorrência em homens. No presente trabalho $77,08 \%$ dos casos notificados foram em pacientes do sexo masculinos.

Por ser uma zoonose primitiva de florestas a leishmaniose é muito resistente as medidas preventivas. Mas algumas devem ser adotadas. Pessoas que apresentar feridas com difícil cicatrização deverá procurar o Centro de Unidade Básica de Saúde, a proteção individual deverá ser feita através de mosquiteiros simples, telas finas em portas e janelas, adicionarem medidas educacionais em saúde e escolas (Alvarenga et al., 2010).

\section{Conclusões}

Para cada ano explorado houve um crescimento considerável da doença, onde os homens foram mais acometidos do que as mulheres, a prevalência maior ocorreu na zona rural e a baixa escolaridade teve um número bastante relevante. A variável idade e raça/cor, os números foram bastante inconstantes. $\mathrm{Na}$ literatura não há relatos que apontem a relevância desses dados.

O estudo sugere no âmbito da Atenção à saúde, ações Inter setoriais, a fim de potencializar difusão de informações, sobretudo à população da zona rural, no sentido de orientá-las quanto às formas de contágio da doença. Além disso, alerta para o crescimento do número de casos nos anos recentes, alertando, portanto, para o reforço nas estratégias de enfrentamento dessa endemia.

\section{Referências bibliográficas}

Almeida, A. B. P. ., Mendonça, A. J., \& Sousa, V. R. F. (2010). Prevalência e epidemiologia da leishmaniose visceral em cães e humanos, na cidade de Cuiabá, Mato Grosso, Brasil. Ciência Rural, 40(7), 1610-1615.

Alvarenga, D. G., Escalda, P. M. F., Costa, A. S. V., \& Monreal, M. T. F. D. (2010). Leishmaniose 
visceral: estudo retrospectivo de fatores associados à letalidade. Revista Da Sociedade Brasileira de Medicina Tropical, 43(2), 194-197.

Basano, S. A., \& Camargo, L. M. A. (2004). Leishmaniose tegumentar americana: histórico, epidemiologia e perspectivas de controle. Revista Brasileira de Epidemiologia, 7, 328-337.

BRASIL. Ministério Da Saúde. Secretaria de Vigilância em Saúde. Programa Nacional de DST e Aids. (2004). Recomendações para diagnóstico, tratamento e acompanhamento da co-infecçãoLeishmaniaHIV. Brasília.

BRASIL. Ministério Da Saúde. (2010). Manual da vigilância da leishmaniose tegumentar americana. $2^{\circ}$ Edição Atualizada. Ministério da Saúde.

BRASIL. Conselho Nacional de Saúde. Resolução n 466, de 12 de dezembro de 2012. Aprova normas regulamentadoras de pesquisas envolvendo seres humanos. Brasília: Diário Oficial da União, 2013.

FNS. (2010). - Fundação Nacional de Saúde (M. de controle da leishmaniose tegumentar americana (ed.)). Ministério da Saúde.

Gontijo, C. M. F., \& Melo, M. N. (2004). Leishmaniose visceral no Brasil: quadro atual, desfios e perspectivas. Revista Brasileira de Epidemiologia, 7(3), 338-349.

Guerra, J. A. O., Talhari, S., Paes, M. G., Garrido, M., \& Talhari, J. M. (2003). Aspectos clínicos e diagnósticos da leishmaniose tegumentar americana em militares simultaneamente expostos à infecção na Amazônia. Revista Da Sociedade Brasileira de Medicina Tropical, 36(5), 587-590.

Jesus, J. R., \& Araújo, F. A. P. (2007). Leishmaniose tegumentar americana: uma visão da epidemiologia da doença na Região Sul. Clínica Veterinária, 12(71), 82-84.

Lucena, R. V., \& Medeiros, J. S. (2018). Caracterização epidemiológica da leishmaniose visceral humana no Nordeste brasileiro, entre 2010 e 2017. Journal of Biology \& Pharmacy and Agricultural Management, 14(4), 285-298.

Miranda, T. M. de, Malaquias, L. C. C., Escalda, P. M. F., Ramalho, K. C., Coura-Vital, W., Silva, A. R. da, Corrêa-Oliveira, R., \& Reis, A. B. (2011). Estudo descritivo sobre a leishmaniose tegumentar americana na área urbana do Município de Governador Valadares, Estado de Minas Gerais, Brasil. Revista Pan-Amazônica de Saúde, 2(1), 27-35.

Oliveira, A. R., \& Fernandes, C. A. (2014). Focos e fatores associados ao aparecimento de leishmaniose tegumentar americana (lta) e leishmaniose visceral (lv) no Cariri Cearense. Revista Interfaces: Saúde, Humanas e Tecnologia, 2(5).

Pimenta, D. N., Leandro, A., \& Schall, V. T. (2007). A estética do grotesco e a produção audiovisual para a educação em saúde: segregação ou empatia? O caso das leishmanioses no BR. 23(5), 1161-1171.

Rey, L. C., Martins, C. V, Ribeiro, H. B., \& Lima, A. A. M. (2005). Leishmaniose visceral americana (calazar) em crianças hospitalizadas de área endêmica. Jornal de Pediatria, 81(1), 73-78.

Santos, G. P., Sanavria, A., Marzochi, M. C., Santos, E. G., Silva, V. L., Pacheco, R. S., Mouta-Confort, E., Espíndola, C. B., Souza, M. B., \& Ponte, C. S. (2005). Prevalência de infecção canina em áreas endêmicas de leishmaniose tegumentar americana do município de Paracambi, Estado do Rio de Janeiro, no período entre 1992 e 1993. Revista da Sociedade Brasileira de Medicina Tropical, 38, 161-166.

Secretária de Estado de Saúde de Minas Gerais - SESMG. Boletim Epidemiológico, Leishmaniose Visceral Humana, Minas Gerais, 2010-2015. Disponível em: <https://www.saude.mg.gov.br/images/documentos/Boletim\%20Epidemiol\%C3\%B3gico\%20Leish maniose_2016.pdf >. Acesso em: 02 nov. 2019.

Silva, O. A., \& Braga, G. M. S. (2010). Leishmaniose visceral canina no município de São Vicente Férrer, Estado de Pernambuco, Brasil. PUBVET, 4, 879-885.

Ursine, R. L., Paranaíba, L. F., Dias, J. V. L., Morais, H. A., \& Pires, H. H. R. (2016). Aspectos epidemiológicos da Leishmaniose Visceral humana e canina em municípios pertencentes à Superintendência Regional de Saúde de Diamantina, Minas Gerais, Brasil (2007-2012). Tempus Actas de Saúde Coletiva, 10(1), 179-193.

Recebido: 04 de novembro, 2019.

Aprovado: 03 de dezembro, 2019.

Publicado: 21 de abril, 2020.
Licenciamento: Este artigo é publicado na modalidade Acesso Aberto sob a licença Creative Commons Atribuição 4.0 (CC-BY 4.0), a qual permite uso irrestrito, distribuição, reprodução em qualquer meio, desde que o autor e a fonte sejam devidamente creditados. 